COMMUNICATIONS IN

ANALYSIS AND GEOMETRY

Volume 11, Number 4, 721-735, 2003

\title{
Embedded Minimal Ends Asymptotic to the Helicoid
}

\author{
David Hoffman AND John MCCuAN
}

\section{Introduction.}

In [5], the genus one helicoid was constructed and strong evidence was given that it is embedded. The surface has infinite total curvature and contains two orthogonal, intersecting straight lines. Its single end has Weierstrass data modeled on that of the helicoid. It is conformally a punctured disk, on which both $d h$ and $d g / g$ have double poles at the puncture. Moreover, these forms have no residues. (See the next section for details about the Weierstrass representation and the helicoid.) In this paper we study this type of end. Our main result is the following.

Theorem 1. Let E be a complete, minimal annular end that is conformally a punctured disk. Suppose $d g / g$ and $d h$ each have a double pole at the puncture and that dh has no residue. If E contains a vertical ray and a horizontal ray, then a sub-end of $E$ is embedded and is asymptotic to a helicoid.

In particular, the genus one helicoid is embedded outside of a compact set of $\mathbb{R}^{3}$; see Figure 1. Although our initial motivation was to establish this fact, ends with Weierstrass data of the type described in the Theorem 1 are of independent interest. The ends of a complete embedded minimal surface of finite total curvature are well understood (every such end is asymptotic to a catenoid or to a plane), but a similar characterization for embedded ends

\footnotetext{
${ }^{1}$ Hoffman was supported by research grant DE-FG03-95ER25250 of the Applied Mathematical Science subprogram of the Office of Energy Research, U.S. Department of Energy, and by research grants DMS-95-96201 and DMS-0139410 of the National Science Foundation, Division of Mathematical Sciences. Research at MSRI is supported in part by NSF grant DMS-90-22140.

${ }^{2}$ During the writing of this paper, McCuan was supported by a National Science Foundation Postdoctoral Fellowship at MSRI and the University of California, Berkeley. Research at MSRI is supported in part by NSF grant DMS-9701755. He is currently supported by NSF grant DMS-0103848
} 
of infinite total curvature is not yet known. A complete minimal surface of finite total curvature is conformally a compact Riemann surface, punctured in a finite number of points corresponding to the ends of the minimal surface. The holomorphic one form $d h$ and the meromorphic function $g$ extend meromorphically to the compactification. This means that $d g / g$ has, at worst, a simple pole, and the poles of $d h$, if there are any, are restricted to the punctures. In the catenoidal case, $d h$ has a simple pole, which means that on a nonplanar embedded end of finite total curvature, both $d g / g$ and $d h$ have simple poles. If one desires to produce an end of infinite total curvature with the conformal type of an isolated singularity, then $d g / g$ must have a pole of order two or greater.

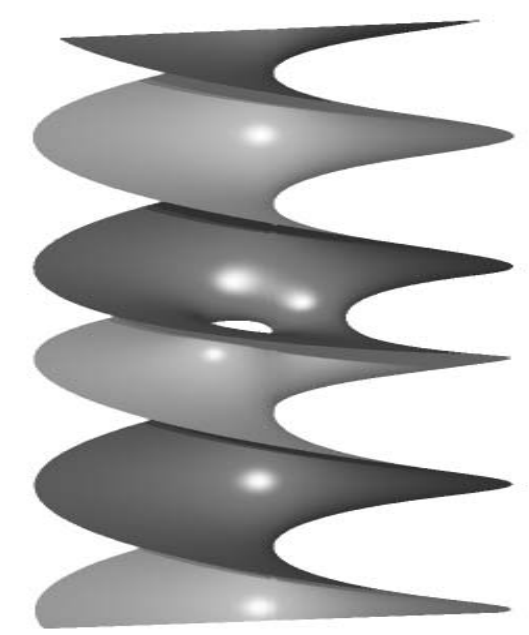

Figure 1: The genus one helicoid.

The end of the helicoid, on which $d g / g$ and $d h$ both have double poles with no residue, is the only classical, embedded annular end of infinite total curvature. It is therefore natural to ask whether embedded, complete, minimal annular ends, on which both $d h$ and $d g / g$ have double poles, must be embedded or asymptotic to a helicoid.

Furthermore, as a consequence of Collin's recent positive resolution of the generalized Nitsche Conjecture [1], a complete embedded minimal surface with finite topology and more than one end must have finite total curvature. This means that a complete, embedded minimal surface with finite topology but infinite total curvature must have just one end. So if we wish to restrict ourselves to looking at an embedded annular end with infinite total 
curvature, which is an end of a complete embedded minimal surface with finite topology, then it is necessarily the only end of the surface. Since $d h$ is holomorphic on the punctured surface, its pole can only occur at the puncture. The sum of the residues of any meromorphic one-form on a compact Riemann surface is zero - a simple consequence of the fact that the union of closed curves around the punctures bounds a compact region that is polefree. In particular, $d h$ must have zero residue at its single pole. Because of this, it is reasonable to assume, as we have in the theorem above, that the ends we study have this property.

The authors wish to thank Harold Rosenberg, Hermann Karcher, and Robert Osserman for useful conversations and advice.

\section{The Weierstrass representation of the Helicoid.}

The helicoid is a simply connected, embedded, ruled minimal surface that is generated by a family of lines, all of which meet a fixed line orthogonally. If we assume that the fixed line is the $x_{3}$-axis, then a horizontal line that sweeps out the helicoid rotates with constant speed (as a function of $x_{3}$ ). From this geometric description, we will derive an analytical one: a Weierstrass representation of the helicoid.

A minimal surface is smooth enough so that the metric induced from $\mathbb{R}^{3}$ has a conformal structure, making it a Riemann surface, $M$. The stereographic projection of the Gauss map, $g: M \rightarrow S^{2}$, is holomorphic, and the coordinate functions are harmonic. Let $h$ be the holomorphic function whose real part is $x_{3}$. Even though $h$ is, in general, only locally defined, $d h$ is globally defined, and one has the Weierstrass representation

$$
X(p)=X\left(p_{0}\right)+\operatorname{Re} \int_{p_{0}}^{p} \Phi, \quad \Phi=\left(\frac{1}{2}\left(g^{-1}-g\right), \frac{i}{2}\left(g^{-1}+g\right), 1\right) d h .
$$

In this representation, the integration takes place on $M$, and the triple $(g, d h, M)$ is referred to as the Weierstrass data.

On the helicoid $h$ is globally defined, since the helicoid is simply connected. Furthermore, from our geometric description, we know that a vertical helix passes through each point of the surface, so $d h$ is never zero. It follows that $h$ is a diffeomorphism of the helicoid with $\mathbb{C}$. We desire the horizontal lines of the helicoid to correspond to horizontal lines in $\mathbb{C}$. To do this, set $z=i h$. Then $d h=-i d z$ and, up to an additive constant,

$$
x_{3}(z)=\operatorname{Re} \int_{z_{0}}^{z} d h=\operatorname{Im}(z),
$$


where $z_{0}$ and $z$ are points in $\mathbb{C}$.

In particular, $X$ maps the lines $\operatorname{Im}(z)=$ constant onto horizontal lines. Since the vertical line meets the horizontal lines orthogonally and the Weierstrass representation (1) is conformal, the preimage of the vertical line on the the surface is a line $\operatorname{Re}(z)=\alpha$ in the $z$-plane. Without loss of generality, we may assume that this line is the imaginary axis, $\operatorname{Re}(z)=0$.

To determine $g$ as a function of $z$, note that along the vertical axis, the normal to the helicoid is always horizontal and rotates at a constant rate: $g(i t)=e^{i c t}$, for some nonzero real constant $c$. By scaling in $\mathbb{R}^{3}$ or by scaling $z$, we may assume, without loss of generality, that $c=1$. Since $g(z)$ is holomorphic, $g(z)=e^{z}$.

To summarize: the Weierstrass data for the helicoid may be written as

$$
g=e^{z}, d h=-i d z \text { on } \mathbb{C} .
$$

We have explicitly written the helicoid as the conformal image of $\mathbb{C}$ (i.e., the Riemann sphere punctured at $\infty$ ). Up to a real translation in $z$ and a rotation in $\mathbb{R}^{3}$, this data is equivalent to

$$
d g / g=d z, d h=-i d z \text { on } \mathbb{C} .
$$

For our purposes, we want to consider it in this form.

\section{Helicoid-type ends: Weierstrass representation.}

We are interested in understanding the behavior of a complete, minimal, annular end, E, that is conformally a punctured disk and has the property that both $d g / g$ and dh have double poles at the puncture. We choose to represent the end on a neighborhood of infinity and so may write $d h=$ $\left(c_{0}+c_{1} / z+\left(1 / z^{2}\right) f(z)\right) d z$, where $f(z)$ is holomorphic and bounded. The assumption that $d h$ has a double pole at infinity implies that $c_{0} \neq 0$. If $\operatorname{Im}\left(c_{1}\right) \neq 0$, then $x_{3}=\operatorname{Re} \int d h$ is not well defined on a neighborhood of infinity. We will make the additional assumption that $c_{1}=0$. As was explained in the introduction, if $E$ is an end of a complete embedded minimal surface of finite topology, this is always the case. Under this assumption, $\zeta(z)=i \int d h$ is a well defined change of variables in a neighborhood of infinity, in terms of which

$$
d h=-i d \zeta
$$

Renaming the variable, we may write $d h$ and $d g / g$ in the following form:

$$
d g / g=\left(a_{0}+a_{1} / z+\left(1 / z^{2}\right) f(z)\right) d z, \quad d h=-i d z,
$$


where $f(z)$ is holomorphic and bounded on a neighborhood of infinity. Note that the Weierstrass representation maps any segment of $\operatorname{Im}(z)=$ constant in the domain onto a curve in a horizontal plane in $\mathbb{R}^{3}$.

We will prove the following propositions.

Proposition 1. Let $E$ be a complete, minimal end with Weierstrass data satisfying (3). Assume that $E$ contains a vertical ray and a horizontal ray. Then $E$ can be represented by Weierstrass data of the form

$$
d g / g=\left(r_{0}+\left(1 / z^{2}\right) f(z)\right) d z, \quad d h=-i d z,
$$

where

$$
f(z)=\sum_{k=1}^{\infty} r_{2 k} z^{2-2 k} .
$$

The coefficients $r_{2 k}, k=0,1,2, \ldots$ are real, and must satisfy the condition

$$
\operatorname{Res}_{z=0}\left(\prod_{k=0}^{\infty} e^{c_{k} z^{1-2 k}}\right)=0,
$$

$c_{k}=r_{2 k} /(1-2 k)$.

Conversely, suppose $f$ is a bounded analytic function on a neighborhood of $\infty$, whose series representation (5) has real coefficients satisfying (6). Then the condition on the Weierstrass data (4) determines a minimal end containing vertical and horizontal rays, and that end is the unique end with these properties.

Proposition 2. Any end of the type described in Proposition 1 is embedded and asymptotic to a helicoid.

\section{The proof of Proposition 1.}

As noted above, the level curves of $E$ are the images under $X$ of lines $\operatorname{Im} z=$ constant in $\mathbb{C}$. After a purely imaginary translation in $z$, we may assume without loss of generality that the horizontal ray is the image of a portion of the real axis $t \geq \rho$ (or $t \leq-\rho$ ) for some $\rho>0$. On the other hand, the second fundamental form for $E$, as noted in $[3,(2.12)]$, is given by

$$
\langle S(v), v\rangle=\operatorname{Re}\left\{\frac{d g}{g}(v) d h(v)\right\}
$$

where $v$ is a tangent vector to $E$. In particular, along $t \geq \rho$ (or $t \leq-\rho$ ), whose image is a line and hence an asymptotic curve, we have that $(d g / g) d h$ 
is purely imaginary. Substituting from (3), this means that

$$
a_{0}+a_{1} / t+\left(1 / t^{2}\right) f(t) \in \mathbb{R} .
$$

On the other hand, $f(z)$ may be expressed as

$$
f(z)=\sum_{j=2}^{\infty} a_{j} z^{2-j} .
$$

In order for (7) to hold for all large $t$, it must be the case that all the $a_{j}, j \geq 0$, are real.

Through each point on the vertical ray $v$ passes a level curve of $x_{3}$ orthogonal to $v$. Since $X$ is conformal and the level curves are the images of the lines $\operatorname{Im} z=$ constant, it follows that $v$ is the image of a ray $\alpha+i t$ for $t \geq \tilde{\rho}$ (or $t \leq-\tilde{\rho})$. By a purely real translation in $z$, we may assume that $\alpha=0$. The condition for an asymptotic curve then yields

$$
\sum_{j=0}^{\infty} a_{j}(i t)^{-j} \in \mathbb{R}
$$

Hence, $a_{j}=0$ for $j$ odd. It follows from this that (4) and (5) are satisfied, and the coefficients are real. Because of this, we will write $r_{k}:=a_{k}$.

Next we integrate the expression for $d g / g$ given in (4) to obtain

$$
g=e^{c} \prod_{k=0}^{\infty} e^{c_{k} z^{1-2 k}} \equiv: A G(z),
$$

where $A=e^{c}, c$ being the constant of integration, and $c_{k}=r_{2 k} /(1-2 k)$.

The residue condition (6) is precisely the requirement that

$$
\int_{\gamma} G(z) d z=0
$$

for $\gamma$ a circle centered at 0 .

Note that $G(z)$ satisfies $G(\bar{z})=\overline{G(z)}$, and $G(-z)=1 / G(z)$. Consequently,

$$
\overline{\int_{\gamma} G(z) d z}=\int_{\gamma} \frac{1}{G(z)} d z=-\int_{\gamma} G(z) d z .
$$

On the other hand, the Weierstrass representation (1) yields a well defined immersion on a neighborhood of infinity provided

$$
\operatorname{Re} \int_{\gamma}\left(g^{-1}-g\right) d h=\operatorname{Re} i \int_{\gamma}\left(g^{-1}+g\right) d h=0
$$


for a sufficiently large circle $\gamma$ centered at $0 \in \mathbb{C}$. This is equivalent to

$$
\int_{\gamma} g^{-1} d h=\overline{\int_{\gamma} g d h}
$$

or

$$
\int_{\gamma} g^{-1} d z+\overline{\int_{\gamma} g d z}=0
$$

since $d h=-i d z$. Substituting into this identity the expression for $g$ from (8) and using (9), we obtain

$$
\left(\bar{A}+\frac{1}{A}\right) \int_{\gamma} G(z) d z=0
$$

This equation can by satisfied if and only if $\int_{\gamma} G(z) d z=0$, which, as noted, establishes (6).

On the other hand, Weierstrass data satisfying (4) and (5) will produce a well defined immersion (1) provided (6) holds. For any choice of $A$, such an immersion always contains a horizontal ray. To see this, we use the formula for the planar curvature of the level curves of a minimal surface expressed in terms of a Weierstrass representation with $d h=-i d z$ (cf. [4]):

$$
\kappa=\operatorname{Im}\left(\frac{g^{\prime}}{g}\right)\left(|g|+|g|^{-1}\right)^{-1} .
$$

From (8) it is clear that $g^{\prime}(t) / g(t)$ is real for $t \in \mathbb{R}$. Hence $\kappa \equiv 0$ on the image of any segment of the real axis, and it follows that the image of $|t| \geq \rho$ is a pair of horizontal rays.

On the imaginary axis, the expression for the Gauss map (8) is unitary provided $|A|=1$, in which case the image of any segment of the imaginary axis is a vertical line segment.

Remark 1. The lines $V$ and $H$ that contain the vertical and the horizontal rays must intersect. If they do not, we can produce, by Schwarz reflection about $V, H$, and their images, an infinite sequence of disjoint vertical and horizontal lines, each of which contains a ray in the end. The images of $H$ all lie in the same horizontal plane, forcing all their preimages in $\mathbb{C}$ to lie on a line of the form $\operatorname{Im}(z)=$ constant. By completeness, such a line can contain the preimage of at most two disjoint rays.

Applying rotations around the lines $V$ and $H$ above, we see that $E$ must contain both "ends" of $H$ and of $V$. 
Different choices of the purely imaginary constant of integration $c$ (i.e., of the unitary constant $A$ ) lead to rigid rotations of the same end about the vertical ray described above. We will show that - except for the Weierstrass data that gives the helicoid - if $A$ is not unitary, then any immersion defined by $(1,4,5)$ contains no vertical ray.

Suppose we have Weierstrass data in this form, defined in a neighborhood $\mathcal{O}$ of infinity. We have shown above that the image of $\mathbb{R} \cap \mathcal{O}$ lies on a horizontal line. The Gauss map $g=A G$ in (8) is unitary on $i \mathbb{R} \cap \mathcal{O}$ if and only if $A$ is unitary there. We have seen above that such a choice of $A$ produces an end containing two rays on a single vertical line, and that different unitary choices of $A$ produce surfaces that differ by a rotation about a vertical axis.

What happens if we choose $|A| \neq 1$ ? For the end to contain vertical rays, there must be a line $L_{\alpha}=\alpha+i t \subset \mathbb{C}$ such that $g$ is unitary on $L_{\alpha} \cap \mathcal{O}$, i.e. $|G|=1 /|A|$. From the definition of $G$ in (8), this is in equivalent to the requirement that $c_{0} z+H(z)$ has constant real part on $L_{\alpha}$, where

$$
H(z)=\sum_{k=1}^{\infty} c_{k} z^{1-2 k}
$$

Since $H(z) \rightarrow 0$ as $z \rightarrow \infty$ and $\operatorname{Re}\left\{c_{0} z\right\} \equiv c_{0} \alpha$ on $L_{\alpha}$, our requirement is in fact the geometric condition that $H\left(L_{\alpha} \cap \mathcal{O}\right) \subset i \mathbb{R}$

Inversion $z \rightarrow 1 / z$ produces the function $h(z):=H(1 / z)=$ $\sum_{k=1}^{\infty} c_{k} z^{2 k-1}$, holomorphic in a neighborhood $\mathcal{O}^{\prime}$ of 0 , whose series expansion is odd with real coefficients. In particular, $h\left(i \mathbb{R} \cap \mathcal{O}^{\prime}\right) \subset i \mathbb{R}$ and $h\left(C_{\alpha} \cap \mathcal{O}^{\prime}\right) \subset i \mathbb{R}$, where $C_{\alpha}$ is the circle produced by inverting $L_{\alpha}$. Note that $C_{\alpha}$ is tangent to $i \mathbb{R}$ at 0 , yet they are both mapped into $i \mathbb{R}$ by the holomorphic map $h(z)$. This is impossible unless $h(z) \equiv 0$, i.e. $H(z) \equiv 0$ and $g(z)=A e^{c_{0} z}$, the Gauss map of the helicoid. It can be put into the standard form of $\S 1$ by the simple change of variables $\zeta=z+c_{0}^{-1} \ln |A|$.

Remark 2. The arguments in the next section rely on the presence of a vertical ray in the surface. It should be noted, however, that if one chooses a non-unitary value of $A$, then $X$ given by (1),(4) is a well defined immersion containing a horizontal ray. It may be the case that such an end is asymptotic to a helicoid, but the techniques of the present paper do not apply.

Remark 3. The condition (6) evidently restricts the coefficients, $c_{j}$. Some indication of the nature of this restriction can be obtained by considering a simple case. Assume that $c_{0}=1$ and $c_{k}=0$ for $k>1$. What are the possible 
values of $c_{1}=a \neq 0$, subject to the condition

$$
\operatorname{Res}_{z=0}\left(e^{z+\frac{a}{z}}\right)=0 ?
$$

Expanding the exponentials in series and setting the coefficient of $1 / z$ equal to zero, we obtain

$$
\sum_{j=0}^{\infty} \frac{(-1)^{j}(-a)^{j}}{j !(j+1) !}=\frac{1}{\sqrt{-a}} J_{1}(2 \sqrt{-a})=0
$$

where $J_{1}$ is the first Bessel function [8, pp. 534-535]. In particular, there is an unbounded sequence of values, a, satisfying the residue condition and yielding ends of the type described in Propositions 1 and 2. One member of this family of ends is illustrated in Figure 2. We will return to this simple class of ends at the end of the next section.

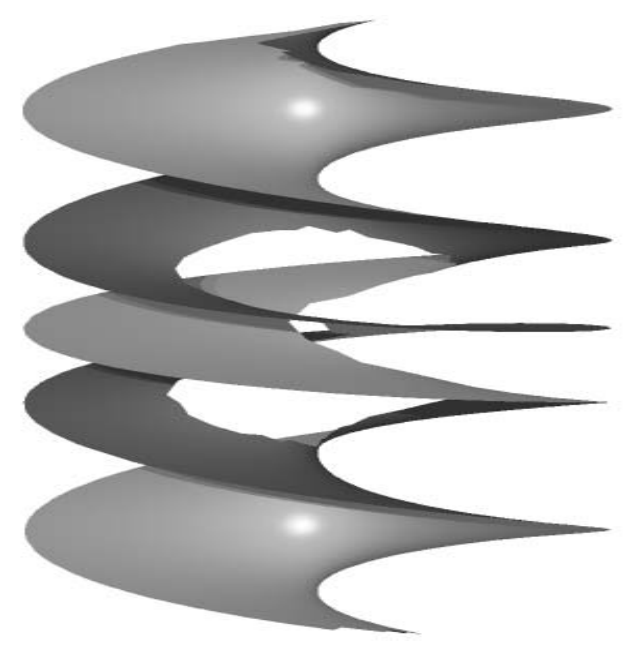

Figure 2: The end with Gauss map $\exp (z+a / z)$ where $a$ is determined by the first zero of the first Bessel function.

\section{The Proof of Proposition 2.}

We assume that our end $E$ is defined on a neighborhood of $\infty$ by Weierstrass data of the form (1),(4). Without loss of generality, we may assume that the constant of integration $c$ is zero and - after scaling if necessary - that $c_{0}=1$. 
We then have

$$
g=e^{z} \prod_{k=1}^{\infty} e^{c_{k} z^{1-2 k}} .
$$

Also, we may restrict attention to a subend of the form $X(\mathcal{D})$ where $\mathcal{D}=$ $\{z=t+i \alpha:|\alpha|>A$ or $|t|>T\}$. We will show that if $A$ and $T$ are large enough, then the level curves $\gamma_{\alpha}(t) \equiv X(t+i \alpha)$, are embedded. Note that for $|\alpha|>A, \gamma_{\alpha}(t)$ is defined for all $t$, while for $|\alpha| \leq A, \gamma_{\alpha}(t)$ consists of two curves $(t>T$ or $t<-T)$.

\section{The End is Embedded.}

We first obtain a general estimate on the curvature of the level curves. We may express the curvature of $\gamma_{\alpha}$ in terms of the Gauss map. From (11), we have

$$
\kappa_{\alpha}(t)=\operatorname{Im}\left(\frac{g^{\prime}}{g}\right)\left(|g|+|g|^{-1}\right)^{-1}
$$

valid for an immersed minimal surface with Gauss map $g$ and $d h=-i d z$; the right-hand side is evaluated at $z=t+i \alpha$. In our case, it is convenient to use the relation $g^{\prime} / g=-i(d g / g) / d h$ to write (using (4) and (5))

$$
\begin{aligned}
\left(|g|+|g|^{-1}\right) \kappa_{\alpha}(t) & =-\operatorname{Re}\left(\frac{d g}{g} / d h\right) \\
& =-\operatorname{Re}\left(i\left(r_{0}+\sum_{k=1}^{\infty} r_{2 k} z^{-2 k}\right)\right) \\
& =\operatorname{Im} \sum_{k=1}^{\infty} r_{2 k} z^{-2 k} .
\end{aligned}
$$

The metric on $X(\mathcal{D})$ is given by $d s=(1 / 4)\left(|g|+|g|^{-1}\right)|d z|$. Hence, along $\gamma_{\alpha}(t)=X(t+i \alpha)$

$$
4\left|\kappa_{\alpha}\right| \frac{d s}{d t} \leq\left|\sum_{k=1}^{\infty} r_{2 k} z^{-2 k}\right| \leq\left(\sum_{k=1}^{\infty} \frac{\left|r_{2 k}\right|}{|z|^{2(k-1)}}\right) \frac{1}{|z|^{2}} .
$$

We assume, without loss of generality, that $A$ and $T$ are large enough so that the series $\sum r_{2 k} z^{2-2 k}$ is absolutely convergent for all $z \in \mathcal{D}$. All values of $\sum\left|r_{2 k} z^{2-2 k}\right|$ for $z \in \mathcal{D}$ are clearly bounded by some $S>0$.

The total absolute curvature of $\gamma_{\alpha}$ is estimated by:

$$
\int_{\gamma_{\alpha}}\left|\kappa_{\alpha}(s)\right| d s \leq \frac{S}{4} \int \frac{d t}{t^{2}+\alpha^{2}} \leq \frac{S \pi}{4 \alpha} .
$$


In particular, when $|\alpha|>S / 4$, the total absolute curvature is strictly less that $\pi$. Hence, when $|\alpha| \geq A_{1}=\max (A, S / 4), \gamma_{\alpha}$ is an embedding of $\mathbb{R}$.

For $|\alpha| \leq A_{1}$, we can apply the first inequality in (13) to show that, for some $T_{1}>T, \gamma_{\alpha}$ is an embedding of $t>T_{1}$. In fact,

$$
\int_{T_{1}}^{\infty}\left|\kappa_{\alpha}(t)\right| \frac{d s}{d t} d t \leq \frac{S}{4} \int_{T_{1}}^{\infty} \frac{d t}{t^{2}+\alpha^{2}} \leq \frac{S}{4} \int_{T_{1}}^{\infty} \frac{d t}{t^{2}}=\frac{S}{4 T_{1}}<\pi
$$

for $T_{1}>\max \{T, S / 4 \pi\}$.

By symmetry $\gamma_{\alpha}(-t), t>T_{1}$ is also an embedded curve. In order to see that $\left\{\gamma_{\alpha}(t)\right\}$ and $\left\{\gamma_{\alpha}(-t)\right\}, t>T_{1}$ are disjoint for $T_{1}$ large enough, we derive a general asymptotic expression (15) for the direction of $\gamma_{\alpha}^{\prime}$, which will also be needed to understand the asymptotic behavior of $E$.

Since $\gamma_{\alpha}$ is a curve in the plane $\left\{x_{3}=\alpha\right\}$, there is a natural projection of $\gamma_{\alpha}$ into $\mathbb{C}$ by the map $\left(x_{1}, x_{2}, \alpha\right) \mapsto x_{1}+i x_{2}$. It causes no confusion to refer to the resulting "complex version" of $\gamma_{\alpha}$ by the same name. As such, the tangent vector $\gamma_{\alpha}^{\prime}$ is a complex number whose argument expresses the direction of the tangent to the level curve $\gamma_{\alpha}$. Moreover, $\arg \gamma_{\alpha}^{\prime}$ can be computed explicitly as follows:

The horizontal projection of the Gauss map of $X(\mathcal{D})$ at $z=t+i \alpha$ is a normal to the plane curve $\gamma_{\alpha}$ at $\gamma_{\alpha}(t)$. Thus, $i g /|g|$ is a unit tangent vector to $\gamma_{\alpha}(t)$, whose direction is given by $\arg (i g)$. In our case,

$$
\arg (g)=\alpha+\operatorname{Im} F(z),
$$

where $F(z)=\sum_{k=1}^{\infty} c_{k} z^{1-2 k}$. We may assume, without loss of generality, that $A$ and $T$ have been chosen so that $\sum\left|c_{k} z^{2-2 k}\right|<C<\infty$ for $z \in \mathcal{D}$. In this way,

$$
|\operatorname{Im} F(z)| \leq|z|^{-1} \sum_{k=0}^{\infty}\left|c_{k+1}\right||z|^{-2 k} \leq \frac{C}{|z|} .
$$

Combining these remarks, we obtain

$$
\arg \gamma_{\alpha}^{\prime}(t)=\frac{\pi}{2}+\alpha+\mathcal{O}\left(\frac{1}{|z|}\right)=\frac{\pi}{2}+\alpha+\mathcal{O}\left(\left|t^{2}+\alpha^{2}\right|^{-1 / 2}\right),
$$

where $\mathcal{O}(1 /|z|) \leq C /|z|$, with $C$ independent of $\alpha$ and $t$.

In applying (15) it will be convenient to consider certain (large) neighborhoods about (the ends of) the lines ruling the helicoid. Let $L_{\alpha}$ be the line through $0 \in \mathbb{R}^{2}$ containing the direction $\vec{r}_{\alpha}=(\sin \alpha, \cos \alpha, 0)$, and let $l_{\alpha}$ be the ray $\left\{t \vec{r}_{\alpha}: t>0\right\}$. For a fixed $\epsilon>0$, let $N_{\alpha}$ be the neighborhood

$$
\bigcup_{|\beta-\alpha|<\epsilon} l_{\beta}
$$


about $l_{\alpha}$. Finally, given any set $S$ in $\mathbb{R}^{2}$ and an angle $\alpha$ that will be evident from the context, we will denote by $\hat{S}$ the lifted set $\left\{(p, \alpha) \in \mathbb{R}^{3}: p \in S\right\}$.

We return now to our original use of $\gamma_{\alpha}$ to denote the level curve at height $\alpha$ in $\mathbb{R}^{3}$ - except where the context dictates otherwise. From the uniform bound in (15) it is straightforward to show the following:

Lemma 1. For any $\epsilon>0$, there is some $T>0$, independent of $\alpha$, such that $\gamma_{\alpha}(t) \in \hat{N}_{\alpha}$ for all $t>T$.

By symmetry, $\gamma_{\alpha}(-t) \in \hat{N}_{\alpha+\pi}$ for $t$ large enough. Since $\hat{N}_{\alpha+\pi}$ and $\hat{N}_{\alpha}$ are disjoint, it is clear that the ends of each level curve are disjoint.

\section{The end is asymptotic to a helicoid.}

We noted in $\S 3$ that, after a rigid translation of $E$ in $\mathbb{R}^{3}$ if necessary, we can assume that $X(i \alpha)=(0,0, \alpha)$ is the point on the standard helicoid with Weierstrass data (2). It is to this particular helicoid that we will show $E$ is asymptotic.

The helicoid is ruled by lines $\hat{L_{\alpha}},-\infty<\alpha<\infty$. Let $H_{\epsilon}$ be the $\epsilon$ neighborhood of the helicoid. It intersects the plane $\left\{x_{3}=0\right\}$ in a set large enough to contain $N_{0} \cup N_{\pi}$. See Figure 3 .

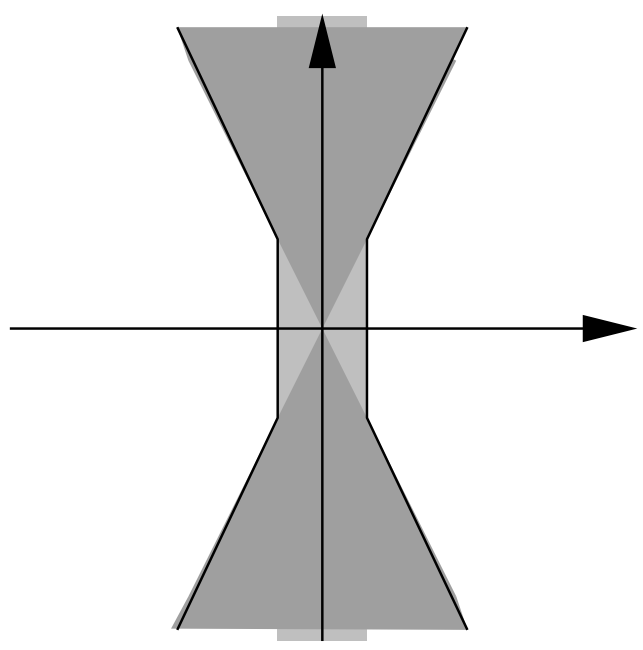

Figure 3: A subset of $H_{\epsilon} \cap\left\{x_{3}=0\right\}$.

Similarly,

$$
\hat{N}_{\alpha} \cup \hat{N}_{\alpha+\pi} \subset H_{\epsilon} \cap\left\{x_{3}=\alpha\right\} .
$$


Note that a small neighborhood of the helicoid in $\mathbb{R}^{3}$ contains quite large neighborhoods of the ruling lines in each plane $\left\{x_{3}=\alpha\right\}$. We have already shown in Lemma 1 that, for $|t|$ large enough, $X(t+i \alpha) \in H_{\epsilon}$. Let $T_{2}>T_{1}$ be such that $|t|>T_{2}$ satisfies this condition. We need only consider $\gamma_{\alpha}(t)$ for $|\alpha|>A_{1}$ and $|t| \leq T_{2}$.

By (15), there is some $A_{2}>A_{1}$ such that for $|\alpha|>A_{2}$ we have

$$
\left|\arg \gamma_{\alpha}^{\prime}(t)-(\alpha+\pi / 2)\right|<\epsilon
$$

Combining this with our normalization $X(i \alpha)=\gamma_{\alpha}(0)=(0,0, \alpha)$, we see that $\gamma_{\alpha}(t) \in \hat{N}_{\alpha} \subset H_{\epsilon}$ for $\alpha>A_{2}$. Symmetry requires, as above, that $\gamma_{\alpha}(-t) \in \hat{N}_{\alpha+\pi}$. This establishes that the end is in $H_{\epsilon}$.

Remark 4. It cannot be expected that the level curves of the ends described by Theorem 1 are asymptotic to the lines ruling the helicoid or, in fact, to any lines. If, for example, $c_{1}=a$ where $J_{1}(2 \sqrt{-a})=0$ as in the remark at the end of $\S 3$, we see that

$$
\left\{\begin{array}{l}
g(z)=e^{z+a / z} \\
d h=-i d z
\end{array}\right.
$$

gives rise to an end $E$ with the following property: If $\hat{E}$ is obtained from $E$ by a clockwise rotation about its vertical axis by an angle $\alpha$, i.e., $\hat{g}=e^{-i \alpha} g(z)$, then in the plane $\left\{x_{3}=\alpha\right\}$ we have $\arg \hat{\gamma}_{\alpha}(t) \rightarrow \pi / 2$, but up to an additive constant

$$
\begin{aligned}
\hat{x}_{1}(t) & =\operatorname{Re} \int_{i \alpha}^{i \alpha+t} \frac{1}{2}\left(\frac{1}{\hat{g}}-\hat{g}\right) d h \\
& =-\operatorname{Im} \int_{0}^{t} \sinh \left(t+\frac{a}{i \alpha+t}\right) d t \\
& =\int_{0}^{t} \sin \left(\frac{\alpha a}{\alpha^{2}+t^{2}}\right) \cosh \left(t+\frac{a}{\alpha^{2}+t^{2}} t\right) d t .
\end{aligned}
$$

Straightforward estimation leads to values $T, C>0$ such that for $t>T$,

$$
\int_{T}^{t} \sin \left(\frac{\alpha a}{\alpha^{2}+t^{2}}\right) \cosh \left(t+\frac{a}{\alpha^{2}+t^{2}} t\right) d t \leq-C \int_{T}^{t} \frac{1}{t^{2}} e^{t} d t .
$$

Since the indefinite integral $\int_{T}^{\infty} t^{-2} e^{t} d t=\infty$, we see that $\hat{x}_{1}$ is unbounded. Therefore, $\hat{\gamma}_{\alpha}$ is not asymptotic to any line, and neither is its rigid rotation $\gamma_{\alpha}$. 


\section{Authors' note}

Years having passed between the acceptance of this paper and its appearance, a short note is warranted about progress directly related to the research presented here. In [2], Hauswirth, Perez and Romon investigated ends of a complete embedded minimal surface in $R^{3}$ of finite type and infinite total curvature. They gave conditions-complementary to those derived here - for such an end to be asymptotic to a helicoid. Under slightly different conditions, they extend the results of this paper. In [7], Hoffman and Wei derived Weierstrass data for a family of screw-motion-invariant genus one helicoids that contain the singly periodic genus-one helicoid, whose existence and embeddedness was proved in [6]. They gave strong computational evidence that this family exists, that its members are all embedded surfaces, and that they limit to an embedded surface of genus one with one end satisfying the hypotheses of Theorem 1 . Such a surface would be an embedded surface of genus one with a single end asympotic to the helicoid. Weber, Wolf and Hoffman, [9] have proved that this continuous family exists and, as predicted, does limit to an embedded genus-one helicoid, the sort of surface whose existence in proved in [5].

\section{References.}

[1] P. Collin. Topologie et courboure des surfaces minimales proprement plongées de $\mathbf{R}^{\mathbf{3}}$. Ann. of Math., 145:1-31, 1997.

[2] L. Hauswirth, J. Pérez, and P. Romon. Embedded minimals ends of finite type. Trans. A.M.S., 353(4):1335, 2001.

[3] D. Hoffman and H. Karcher. Complete embedded minimal surfaces of finite total curvature. In R. Osserman, editor, Encyclopedia of Mathematics: Minimal Surfaces, pages 5-90. Springer, 1997.

[4] D. Hoffman, H. Karcher, and H. Rosenberg. Embedded minimal annuli in $\mathbf{R}^{3}$ bounded by a pair of straight lines. Comment. Math. Helvetici, 66:599-617, 1991.

[5] D. Hoffman, H. Karcher, and F. Wei. The genus one helicoid and the minimal surfaces that led to its discovery. In K. Uhlenbeck, editor, Global Analysis and Modern Mathematics, pages 119-170. Publish or Perish, 1993. 
[6] D. Hoffman, H. Karcher, and F. Wei. The singly periodic genus-one helicoid. Comment. Math. Helvetici, 74:248-279, 1999.

[7] D. Hoffman and F. Wei. Deforming the singly periodic genus-one helicoid. Experimental Mathematics, 11:207-218, 2002.

[8] Samuel M. Selby, editor. CRC Standard Mathematical Tables, 23rd edition. CRC Press, Cleveland, 1975.

[9] M.Weber, D.Hoffman, and M.Wolf, An embedded genus-one helicoid as the limit of periodic embedded minimal surfaces. Preprint, 2002.

DAVID HOFFMAN

Mathematical Sciences Research Institute

17 Gauss WAY

BERKELEY CA 94720

david@msri.org

JOHN MCCUAN

School of Mathematics

Georgia Institute of Technology

Atlanta, GA 30332-0180

mccuan@math.gatech.edu

Received August 27, 1999. 